\title{
Redes de colaboración científica en los estudios territoriales
}

\author{
Yuritzi Becerril-Tinoco. El Colegio Mexiquense A.C., Zinacantepec, México. \\ Rosario Rogel-Salazar. Universidad Autónoma del Estado de México, Toluca, México
}

RESUMEN | Se presenta una investigación sobre la colaboración científica en el área de los estudios territoriales. Se retoma el enfoque de la teoría de redes sociales y de sistemas complejos, ampliamente desarrollado por Loet Leydesdorff. A través de un análisis estadístico basado en fuentes como Redalyc, Scopus, Web of Science y Scielo, se muestran las pautas de colaboración en el campo de estudios territoriales y se señala que existe relación entre el incremento de la literatura de este campo y la publicación en colaboración. Finalmente se presentan los indicadores generales de producción científica de una revista de esta área temática, Economía, Sociedad y Territorio, y se contrastan los resultados con la tendencia general en América Latina. La investigación muestra que los estudios urbano-regionales constituyen un campo altamente colaborativo.

PALABRAS CLAVE | colaboración científica, estudios urbano-regionales, producción científica, revistas académicas, redes sociales.

ABSTRACT | Research on scientific collaboration in the context of territorial studies is presented, utilizing the approach of social networks and complex systems theories, which were thoroughly developed by Loet Leydesdorff. By means of an analysis based on sources such as Redalyc, Scopus, Web of Science and Scielo, patterns of collaboration in the field of territorial studies are reviewed. The results show that there is a relation between the increase in the literature on this field of knowledge and collaboratory publications. Finally, the general indicators regarding the scientific outputs of a journal in this field (Economia, Sociedad $y$ Territorio) are presented, and the results are contrasted with a general tendency that has been defined for Latin America. The research shows that urban-regional studies are a highly collaborative field.

KEYWORDS | scientific collaboration, urban-regional studies, scientific output, academic journals, social networks. 


\section{Introducción}

La presente investigación hace referencia a las redes de colaboración que se generan en el sistema de comunicación científico latinoamericano, en el área de los estudios territoriales.

De acuerdo con la literatura existente, en los estudios sobre colaboración científica se pueden identificar diferentes enfoques. Una línea se basa en análisis bibliométricos que utilizan como fuente de información la base de datos de Web of Science (WoS) de Thompson-Reuters para analizar las relaciones de cocitación: Abrizah, Zainab, Kiran y Raj (2013); Ni, Sugimoto y Cronin (2013). Desde la perspectiva de la sociología de la ciencia y la comunicación: la investigación del Centro de Estudios sobre Ciencia y Tecnología de la Universidad de Leiden (cWTs, 2012); Leydesdorff (2013), Leydesdorff, Park y Wagner (2014). Del grupo Scimago: Miguel, Chinchilla-Rodríguez, González y De Moya-Anegón (2012); Molina, Muñoz y Doménech (2002). En el área de los estudios territoriales: Aguado-López, Rogel-Salazar, Álvarez-Velázquez, Muñoz-Eraso y López-López (2008); Kamalski y Kirby (2012); Link (2008); Pino-Díaz, Jiménez-Contreras, Ruíz-Baños y Bailón-Moreno (2011), Royuela (2012).

\section{El crecimiento de la literatura científica en red}

Como lo documenta la Royal Society, entre los siglos xvir y xx el crecimiento de la literatura científica se dio de manera lineal y exponencial. Actualmente se ha mostrado que la ciencia está experimentando un fenómeno de crecimiento en red, a nivel nacional e internacional. (The Royal Society, 2011). De acuerdo con información de esta misma sociedad científica, actualmente menos del $26 \%$ de las comunicaciones científicas son producto de una sola institución y una tercera parte tiene múltiples nacionalidades en coautoría.

La tendencia a participar y colaborar en red responde a la consolidación de una estructura científica y a la rapidez de la diseminación de un paradigma de producción adoptado. Collazo-Reyes, Luna-Morales y Vélez Cuartas (2010) señalan que a "mayor consolidación [de la disciplina], mayores son los grados de estandarización de las prácticas y metodologías de investigación” (s/p). La estandarización del modelo de comunicación científica ha sido más exitosamente incorporada por las disciplinas de ciencia exacta de occidente y se ha expandido lentamente hacia las ciencias sociales y los países periféricos, en una dinámica que actualmente es altamente favorecida por el contexto digital (Leydesdorff, 2003).

Se han identificado dos tendencias: por un lado, se ha mostrado que las naciones desarrolladas publican más en colaboración que los países periféricos. Y en segundo lugar, se ha visto que el crecimiento del número de publicaciones está acompañado del aumento en el número de trabajos publicados en coautoría. Es decir, que la investigación está creciendo en forma de red (The Royal Society, 2011). 
GRÁfICO I Producción científica en colaboración y sin colaboración 2005-2011, en revistas de acceso abierto, por número de documentos
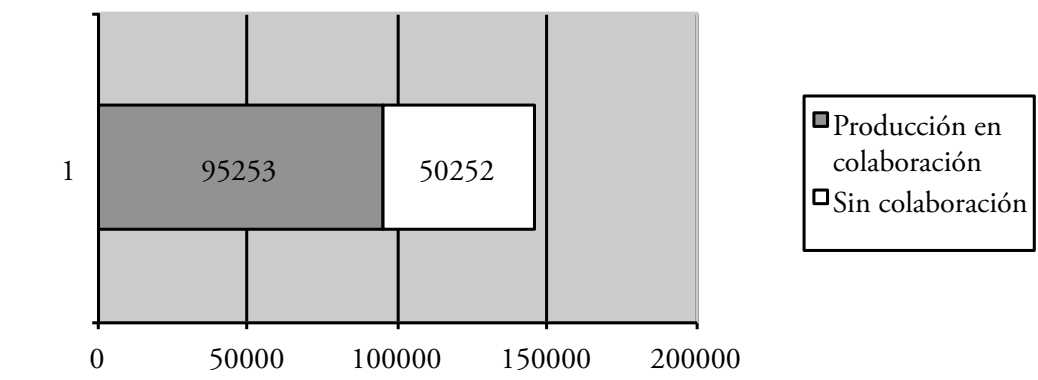

FUENTE ELABORACIÓN PROPIA CON BASE EN REDALYC (20I3)

\section{El análisis de redes de colaboración científica}

Entre otros métodos estadísticos, señalan Perianes-Rodríguez, Olmeda-Gómez y Moya-Anegón (2008, p. 667), las redes de información o de conocimiento en el mundo científico pueden identificarse a través del análisis de las citaciones y cocitaciones de los trabajos, pero también por la relación entre conceptos. Su análisis da cuenta de la primacía de algunos conceptos, de su evolución o la constitución de fronteras analíticas entre las disciplinas.

El campo de los estudios urbano-territoriales: una caracterización

En los estudios territoriales se puede identificar un cluster especializado y único en estudios urbanos; un cluster relativo a los estudios urbanos publicados en ciencias sociales; y uno más relativo a los estudios urbanos publicados en el campo de las ciencias aplicadas (Kamalski \& Kirby, 2012).

Las revistas de estudios urbano-territoriales albergan investigaciones provenientes tanto del área de las ciencias sociales como de las ciencias aplicadas o exactas. En el WoS-JCR se identifican nueve vínculos fuertes de las revistas de estudios urbanoterritoriales cuyos nodos principales se concentran en los Estudios Ambientales, Economía, y Planeación y Desarrollo, en ese orden de importancia. En SCOPUS, los tres nodos más fuertes, de las quince disciplinas con más vínculos comunicativos registrados, corresponden a Geografía, Planeación y Desarrollo; Sociología y Ciencia Política; y Ciencias Ambientales. En la base de datos Redalyc, igualmente con quince disciplinas en interacción con los estudios urbanos, los nodos más grandes de interrelación se encuentran en los Estudios Territoriales, Geografía Social y Biología (Redalyc, 2013; sJr, 2013; WoS, 2013)

De acuerdo con el estudio de Kamalski y Kirby (2012, p. 5), podemos saber que, en ciencias, estos estudios aparecen relacionados con los temas agua, medioambiente, aire, atmósfera, contaminación, sustentabilidad, clima, sistemas de información geográfica (SIG), transporte o agricultura; en ciencias sociales, con historia o estudios de género; y en estudios urbanos, con economía urbana, gobernanza, cambio social, renovación urbana, sociedad urbana, política urbana, educación; mientras que los 
temas comunes a los tres campos son área urbana, planeación urbana, urbanización, desarrollo urbano; los estudios sobre vivienda y área metropolitana se discuten sobre todo en el marco de las ciencias sociales y los estudios urbanos.

Un mapeo construido por Bornmann y Leydesdorff (2013) muestra la distribución regional de autores que concentran mayor cantidad de artículos citados, los cuales publican desde Europa, algunos países de Asia y Estados Unidos. Según el estudio de Kamalski y Kirby (2012), los países más productivos en esta área son Estados Unidos y Reino Unido, que superan por un porcentaje muy alto al resto de los países europeos y periféricos. Australia, Canadá y Países Bajos son también altamente representativos; y en el área de ciencias figuran China, Alemania, España, Francia, India, Japón, Italia, Brasil.

\section{Los estudios territoriales en América Latina}

En el continente latinoamericano, de acuerdo con Carlos A. de Mattos (2013), el auge de los estudios urbano-regionales sucede hacia la década de los años sesenta, con temas prioritarios en ese momento histórico en el continente americano: la migración campo-ciudad; la urbanización y la correspondiente metropolización de las ciudades; la marginación, segregación y desigualdades sociales y regionales.

En la primera década del siglo xx se crean las primeras revistas de estudios regionales, con una fuerte tendencia hacia los estudios agrarios. El antecedente más lejano lo encontramos en los Anales de la Sociedad Rural Argentina, que se edita desde 1866 en la ciudad de Buenos Aires. Hacia la década de los sesenta, las revistas de investigación acogen temas relativos a la urbanización latinoamericana desde una perspectiva territorial. En 1962, se crean los Cuadernos de la Sociedad Venezolana de Planificación; en 1967, la Revista Mexicana de Planificación; el Centro de Estudios Demográficos y de Desarrollo Urbano de El Colegio de México crea la revista Demografía y Economía en 1967; en 1970 aparece EURE, editada por el Instituto de Estudios Urbanos y Territoriales de la Pontificia Universidad Católica de Chile; en São Paulo aparece Espaço \& Debates en 1980 (Latíndex, 2014).

\section{Pautas de colaboración en el área de los estudios territoriales}

A partir del estudio publicado por Royuela (2012), en la década de los noventa, el $52 \%$ de los artículos publicados en revistas mainstream en el área de estudios regionales y economía urbana eran de autoría única (Surinach, Duque, Ramos \& Royuela, 2002, citado en Royuela, 2012); sin embargo, esta tendencia ha cambiado. De acuerdo con los resultados de la investigación del mismo autor, el 67\% de los trabajos discutidos en la conferencia anual de la European Regional Science Association (ERSA) en 2011 fueron elaborados en coautoría ${ }^{1}$. Por otra parte, de acuerdo con nuestra investigación, la estadística de producción científica para el área de los estudios territoriales en América Latina muestra que, en el periodo de 2005 a 2011, el 40,7\% de los trabajos fueron publicados en colaboración. Esto representa un cambio en la forma de producir conocimiento en el continente, ya que en un

1 De los documentos presentados, 34\% lo fueron de autoría única; 33\% fueron escritos por dos autores, $23 \%$ de los trabajos fueron firmados por tres autores y $10 \%$ se realizaron en coautoría de más de tres autores. 
periodo más largo (1996-2013), la publicación en colaboración es de solo 29,5\%. Cabe resaltar que, en general, para un periodo de cinco años, la colaboración es en mayor medida institucional $(61,5 \%)$, nacional $(27,6 \%)$ mientras que las redes de colaboración entre investigadores e instituciones de diferentes países es aún incipiente (10,9\%) (Redalyc, 2013).

El Mapa I, muestra la forma en que se distribuye la producción total, de distintas regiones del mundo y que fueron publicadas en revistas iberoamericanas de acceso abierto durante el periodo 2005-2010.

El nivel más alto de producción lo presenta Iberoamérica, con 115.926 trabajos en estudios territoriales, seguido de Europa con 5.415 y Estados Unidos con 5.298; Asia, África y Oceanía ocupan los últimos lugares de producción.

Entre los tres primeros lugares de producción se observa que, mientras en Iberoamérica es mayor la producción nacional (institucional y no institucional), y la extranjera no alcanza ni el 50\% de la producción total ni en colaboración, en Europa y Estados Unidos la producción total y la producción en colaboración extranjera es la más elevada.

MAPA I | Producción 2005-2010 por región del mundo en revistas iberoamericanas de acceso abierto

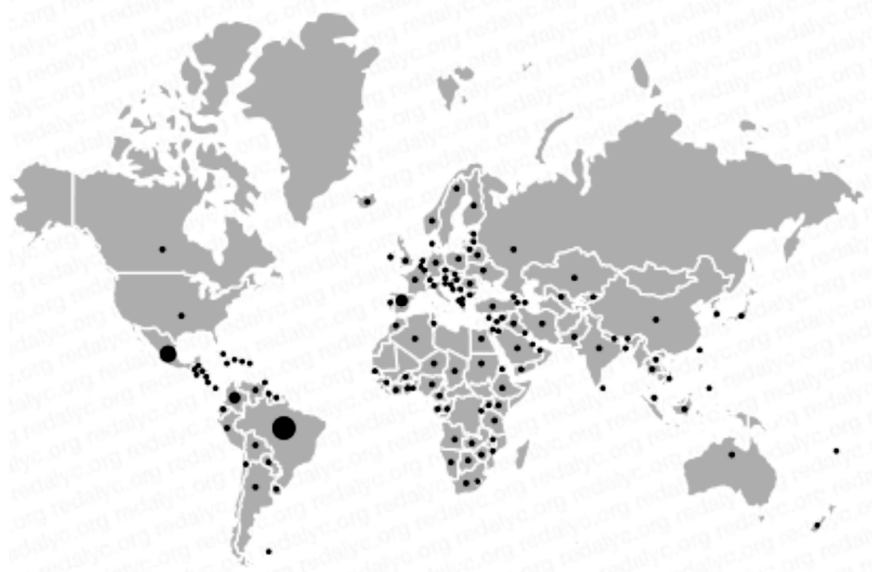

FUENTE REDALYC, 20 I 3 (I DE OCTUBRE DE 2OI3)

De manera general, también se puede advertir que la producción en colaboración en cada una de las regiones (Iberoamérica, Europa, América del Norte, Asia, Oceanía y África) supera el 50\% de la producción total, lo cual revela que las redes de colaboración van en ascenso. Así se observa en el Cuadro 1, donde la P, representa la Producción autoral única y PC la producción en colaboración. 
CUADro I | Producción 2005-2010 por región del mundo en revistas iberoamericanas de acceso abierto

\begin{tabular}{|l|r|l|l|r|}
\hline \multicolumn{2}{|c|}{ AMÉRICA DEL NORTE } & \multicolumn{3}{c|}{ IBEROAMÉRICA } \\
\hline P & 5.238 & P & 115.926 \\
\hline PC & 63.295 & PC & \multicolumn{3}{|c|}{ ÁFRICA } \\
\hline \multicolumn{3}{|c|}{ EUROPA } & P & 75.156 \\
\hline P & 5.415 & PC & 373 \\
\hline PC & 61.830 & P & \multicolumn{2}{c|}{ OCEANÍA } \\
\hline \multicolumn{7}{|c|}{ ASIA } & 1.208 & PC & 212 \\
\hline P & 77.456 & & \\
\hline PC & &
\end{tabular}

P = PRODUCCIÓN AUTORIAL ÚNICA; PC = PRODUCCIÓN EN COLABORACIÓN

FUENTE ELABORACIÓN PROPIA CON BASE EN REDALYC, 20 I 3 (I DE OCTUBRE DE 2OI3)

En el siguiente gráfico se señala la producción en colaboración en trabajos de estudios territoriales en los años del 2005 al 2010. La producción en colaboración registra un aumento anual con diferencias que van de un punto porcentual hasta cinco entre el primer y el último ańo considerado.

GRÁFICO 2 | Artículos en colaboración. Estudios territoriales 2005-2010

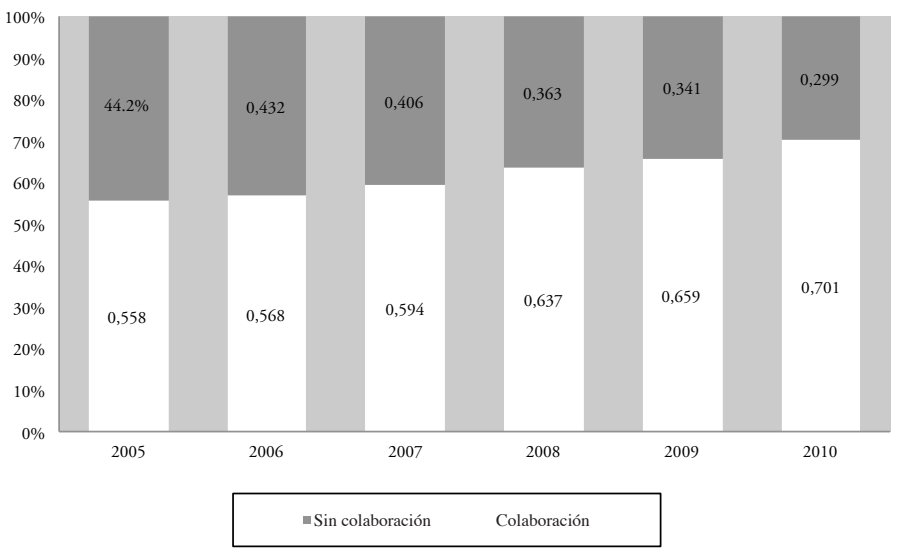

FUENTE REDAlyc, 20 I 3 (CONSUlta I DE OCtubre de 2OI3)

Evolución de la producción en colaboración en los estudios territoriales, 2005-2010 Estadísticas derivadas de la base de datos Redalyc (2013) revelan un aumento de la producción de trabajos en estudios territoriales generados por medio de redes de colaboración entre 2005 y 2010. Los datos analizados corresponden al 62,4\% de la producción total de trabajos en estudios territoriales, es decir, 4.652 publicaciones. De manera general se observa un crecimiento constante de la producción en colaboración. La mayor producción en colaboración corresponde a la producción nacional institucional, le sigue la producción nacional no institucional, mientras que la producción extranjera presenta el menor índice de producción en colaboración durante los cinco ańos considerados. 
CUADRO 2 | Evolución de la producción en colaboración. Estudios territoriales 2005-2010

\begin{tabular}{|c|c|c|c|c|c|c|c|}
\hline \multicolumn{8}{|c|}{ ABSOLUTOS } \\
\hline \multicolumn{2}{|c|}{ Colaboración } & 2005 & 2006 & 2007 & 2008 & 2009 & 2010 \\
\hline \multirow{2}{*}{ Nacional } & Institucional & 312 & 334 & 391 & 439 & 498 & 522 \\
\hline & No institucional & 199 & 232 & 242 & 258 & 279 & 352 \\
\hline \multicolumn{2}{|l|}{ Extranjera } & 73 & 84 & 93 & 101 & 137 & 106 \\
\hline \multicolumn{8}{|c|}{ PORCENTAJES } \\
\hline \multicolumn{2}{|c|}{ Colaboración \% } & 2005 & 2006 & 2007 & 2008 & 2009 & 2010 \\
\hline \multirow{2}{*}{ Nacional } & Institucional & 53,4 & 51,4 & 53,9 & 55 & 54,5 & 53,3 \\
\hline & No institucional & 34,1 & 35,7 & 33,3 & 32,3 & 30,5 & 35,9 \\
\hline \multicolumn{2}{|l|}{ Extranjera } & 12,5 & 12,9 & 12,8 & 12,7 & 15 & 10,8 \\
\hline
\end{tabular}

FUENTE ELABORACIÓN PROPIA CON BASE EN REDALYC (LABORATORIO DE CIENCIOMETRÍA REDALYCFRACTAL, 2OI3)

\section{El caso de la revista Economía, Sociedad y Territorio. Indicadores generales de producción científica}

En el siguiente apartado se analizan los patrones de colaboración en la revista Economí, Sociedad y Territorio (est).

El Cuadro 3 muestra el grado de internacionalización de la revista, así como el número de trabajos publicados por periodos quinquenales. Mientras que en los primeros cinco ańos de vida de la revista est se muestra un grado de internacionalización del 39,34\%, contra $60,6 \%$ nacionales, en el último periodo esta tendencia se reafirma (el $67,84 \%$ de artículos son nacionales, mientras que el 32,16\% son internacionales).

CUADRO 3 | Indicadores generales de producción científica (frecuencias)

\begin{tabular}{|l|c|c|c|c|c|c|}
\hline \multirow{2}{*}{ PERIODO } & \multirow{2}{*}{ TRABAJOS } & \multicolumn{3}{|c|}{ INSTITUCIONES } & \multicolumn{2}{c|}{ PORCENTAJE } \\
\cline { 3 - 7 } & & NACIONALES & $\begin{array}{c}\text { INTERNACIO- } \\
\text { NALES }\end{array}$ & TOTAL & NACIONALES & $\begin{array}{c}\text { INTERNACIO- } \\
\text { NALES }\end{array}$ \\
\hline $1997-2001$ & 61 & 37 & 24 & 61 & 60,66 & 39,34 \\
\hline $2002-2006$ & 78 & 46 & 32 & 78 & 58,97 & 41,03 \\
\hline $2007-2011$ & 144 & 109 & 35 & 144 & 75,69 & 24,31 \\
\hline Total & 283 & 192 & 91 & 283 & $\mathbf{6 7 , 8 4}$ & 32,16 \\
\hline
\end{tabular}

FUENTE ARCHIVOS DE LA REVISTA est I 997-20 I I

\section{Colaboración institucional, nacional, internacional}

Al contrastar la información con las estadísticas generadas por el Sistema de Información Científica Redalyc, visible en el Cuadro 4, observamos que el promedio de producción autoral de la revista est 2005-2011 nacional (que contempla también la producción institucional) es de 65,6\%, mientras que la producción autoral internacional es de $34,4 \%$. Ambos datos permiten observar que la revista est es un espacio editorial abierto, en la medida en que da prioridad a la investigación 
producida nacional e internacionalmente, que en conjunto suman un 96,4 por ciento.

CUADRO 4 | Perfil general de producción y colaboración 2005-2011. Revista est

\begin{tabular}{|l|c|c|c|c|}
\cline { 2 - 4 } \multicolumn{1}{c|}{} & \multirow{2}{*}{ INTERNACIONAL } & \multicolumn{2}{|c|}{ TOTAL NACIONAL } & \multirow{2}{*}{ TOTAL } \\
\cline { 3 - 4 } & & NACIONAL & INSTITUCIONAL & 100 \\
\hline Producción autoral & 34,4 & 62 & 3,6 & 100 \\
\hline Colaboración & 10 & 34,44 & 55,56 & \multirow{2}{*}{} \\
\hline
\end{tabular}

FUENTE ARCHIVOS REVISTA est I997-20I I

Podemos contrastar los resultados de la revista est con los de las revistas de estudios territoriales indexadas en Redalyc. Se observa que los resultados de est corresponden a una dinámica regional, pues al igual que lo reseńado por Redalyc, apenas el 10,9\% de los trabajos se produce en colaboración entre instituciones de diferentes países.

CUADRO 5 | Perfil general de producción y colaboración 2005-2011. Estudios territoriales

\begin{tabular}{|c|c|c|c|c|}
\hline & \multirow{2}{*}{ INTERNACIONAL } & \multicolumn{2}{|c|}{ TOTAL NACIONAL } & \multirow{2}{*}{ TOTAL } \\
\hline & & NACIONAL & INSTITUCIONAL & \\
\hline Producción & 39,32 & 47,35 & 13,33 & 100 \\
\hline Colaboración & 10,9 & 27,61 & 61,49 & 100 \\
\hline
\end{tabular}

FUeNte REDAlyc, 2013 (CONSUlta I De OCtubre DE 2013)

\section{Patrones de colaboración interinstitucional}

La información normalizada de autores e instituciones de adscripción permite observar que, a lo largo de su trayectoria, la revista est ha creado una red de colaboración con 99 instituciones diferentes. Aunque no todas constituyen verdaderas redes de colaboración continua, sí se puede hablar de un núcleo fuerte de instituciones entre las cuales se colabora. En este caso, la revista est presenta patrones regulares de colaboración con catorce instituciones, que se pueden observar en el Cuadro 6.

CUADRO 6 | Núcleo fuerte de instituciones. Revista est

\begin{tabular}{|l|c|c|c|c|}
\hline \multicolumn{1}{|c|}{ INSTITUCIONES } & I997-200I & 2002-206 & 2007-20I I & TOTAL \\
\hline El Colegio Mexiquense, A.C. & 14 & 7 & 7 & 28 \\
\hline Universidad Autónoma del Estado de México & & 5 & 16 & 21 \\
\hline Universidad Nacional Autónoma de México & 1 & 2 & 16 & 19 \\
\hline El Colegio de México & 8 & 2 & 1 & 11 \\
\hline Colegio de Postgraduados & & & 11 & 11 \\
\hline Universidad de Buenos Aires & 2 & 6 & 2 & 10 \\
\hline El Colegio de la Frontera Norte, A.C. & 2 & 3 & 4 & 9 \\
\hline Universidad de Guadalajara & & 3 & 6 & 9 \\
\hline
\end{tabular}


(continuación)

\begin{tabular}{|l|c|c|c|c|}
\hline \multicolumn{1}{|c|}{ INSTITUCIONES } & I997-200I & 2002-206 & 2007-20I I & TOTAL \\
\hline Universidad Nacional de Colombia & 2 & 5 & 1 & 8 \\
\hline $\begin{array}{l}\text { Instituto Tecnológico y de Estudios Superio- } \\
\text { res de Monterrey }\end{array}$ & & 3 & 5 & 8 \\
\hline Universidade Federal do Rio de Janeiro & 1 & 4 & 1 & 6 \\
\hline $\begin{array}{l}\text { Centro de Investigación y Docencia Econó- } \\
\text { micas, A.C. }\end{array}$ & 3 & 1 & 2 & 6 \\
\hline Universidad Autónoma Metropolitana & 5 & & 1 & 6 \\
\hline Universidad Autónoma de Zacatecas & & 1 & 3 & 4 \\
\hline
\end{tabular}

FUENTE ARCHIVOS DE LA REVISTA EST I 997-20 I I Y REDALYC 2005-20 I I (CONSULTA I DE OCTUBRE DE 2OI3)

Por otro lado, se presenta la colaboración entre países, siendo México, Argentina y Brasil los países desde donde se produce la mayor cantidad de las investigaciones que publica la revista estudiada. Los siguen España, Venezuela y Colombia.

\section{CUADRO 7 | Colaboración entre países. Revista est}

\begin{tabular}{|l|c|c|}
\hline \multicolumn{1}{|c|}{ PAÍs } & CANTIDAD & PORCENTAJE \\
\hline México & 66 & 73,3 \\
\hline Argentina & 7 & 7,8 \\
\hline Brasil & 6 & 6,7 \\
\hline España & 4 & 4,4 \\
\hline Venezuela & 3 & 3,3 \\
\hline Colombia & 2 & 2,2 \\
\hline Canadá & 1 & 1,1 \\
\hline Cuba & 1 & 1,1 \\
\hline
\end{tabular}

FUENTE REDALyC, 2013 (CONSUlta I DE OCtubre DE 2OI3)

\section{MAPA 2 | Producción por países. Revista est}

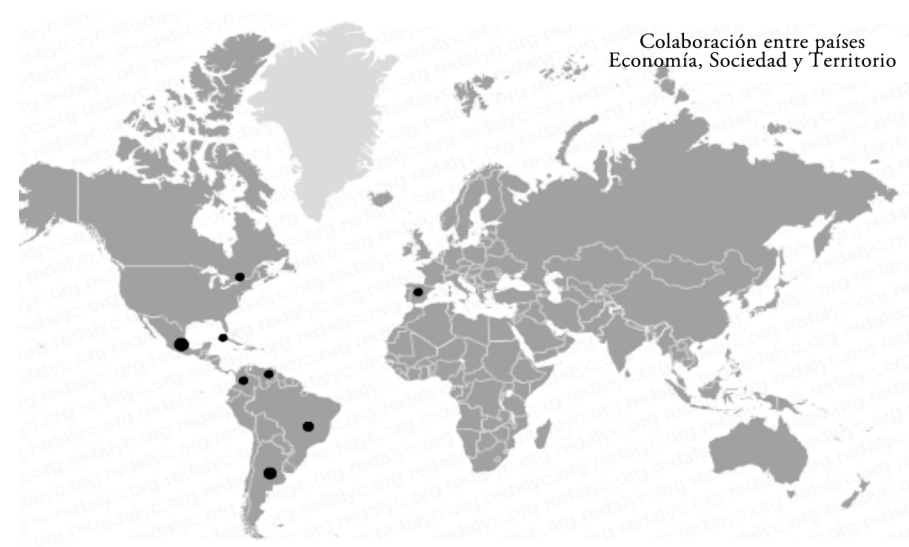

FUENTE REDAlyc, 20 I 3 (CONSUlta I DE OCtubre De 2OI3) 
Observando la estadística correspondiente a los estudios territoriales, vemos que hay marcadas diferencias en este rubro, ya que en general, de acuerdo con el acervo de Redalyc (2013), los países que más colaboran en la publicación de resultados en este campo científico son, en el orden siguiente, Chile, México, España, Colombia, Venezuela y Brasil. Es decir, que una posible política científica para esta revista sería atraer investigaciones originales de estos países.

\section{Estructura colaborativa: la publicación en red}

En el Cuadro 8 se muestra la estructura colaborativa del área Estudios territoriales observada en la revista est, que desde el año 2000 revela una tenue, pero cada vez más sostenida orientación a la publicación en red. Es posible confirmar esta tendencia, observando el número de autores que han participado en la elaboración de un documento durante el periodo 2007-2011, donde se ubican papers firmados hasta por seis y siete autores, que representan poco más del $6 \%$ del total de publicaciones. Si comparamos el desarrollo de la publicación en red de la revista est con la tendencia mundial, observamos una clara correspondencia. Por ejemplo, los resultados dados a conocer por la Royal Society (2011) muestran que entre los años 2000 y 2008, Francia y Alemania duplicaron el número de artículos en coautoría. La matriz de colaboración (Cuadro 8), indica que el porcentaje de trabajos en coautoría en la revista est se ha duplicado en cada periodo señalado, pasando de 18 a $34 \%$ del primer al segundo periodo, y casi duplicando esta cifra en el tercer periodo, es decir de 34 a $63 \%$.

CUADRo 8 | Matriz de relaciones trabajos/número de autores

\begin{tabular}{|c|c|c|c|c|c|c|c|c|c|}
\hline \multirow{2}{*}{ PERIODO } & \multicolumn{6}{|c|}{$\begin{array}{l}\text { NÚMERO DE AUTORES POR } \\
\text { DOCUMENTO }\end{array}$} & \multirow{2}{*}{$\begin{array}{l}\text { TOTAL DE } \\
\text { TRABAJOS }\end{array}$} & \multirow{2}{*}{$\begin{array}{l}\text { TRABAJOS EN } \\
\text { COLABORACIÓN }\end{array}$} & \multirow{2}{*}{$\begin{array}{c}\% \\
\text { TRABAJOS EN } \\
\text { COLABORACIÓN }\end{array}$} \\
\hline & I & 2 & 3 & 4 & 5 A 6 & $>6$ & & & \\
\hline $1997-2001$ & 50 & 9 & 2 & 0 & 0 & 0 & 61 & 11 & 18,03 \\
\hline $2002-2006$ & 51 & 19 & 6 & 0 & 2 & 0 & 78 & 27 & 34,62 \\
\hline $2007-2011$ & 53 & 52 & 19 & 11 & 8 & 1 & 144 & 91 & 63,19 \\
\hline Total & 155 & 82 & 30 & 15 & 10 & 1 & 283 & 138 & 48,76 \\
\hline
\end{tabular}

FUENTE ARCHIVOS DE LA REVISTA EST I 997-20I I

Lo anterior nos permitiría afirmar no solo que la investigación en los estudios territoriales publicados por la revista est está creciendo en red, sino que esta área constituye un campo eminentemente colaborativo, puesto que actualmente más del $90 \%$ de las investigaciones publicadas por esta revista se firman en coautoría.

Los resultados de esta investigación muestran que el campo de los estudios territoriales sigue la pauta global de la ciencia, que indica un crecimiento exponencial de la tendencia colaborativa. Como se observa en el Cuadro 9, Gráfico III, en el primer periodo de estudio, básicamente la investigación era producida en el seno de una sola institución: la cifra que se observa en este rubro representa el $82,2 \%$. Se alcanza un equilibro en el último periodo en la producción de una institución y dos instituciones, que representan 42,7 y $41,9 \%$, respectivamente. La participación 
de tres instituciones muestra en todos los periodos un crecimiento constante, de tal modo que al final del último periodo quinquenal observado, son 19 los documentos en donde participan tres instituciones. En el Gráfico III se observa cómo cae el número de artículos firmados por autores de una sola institución.

\section{GRÁfICO 3 | Crecimiento de los trabajos en colaboración de la revista est}

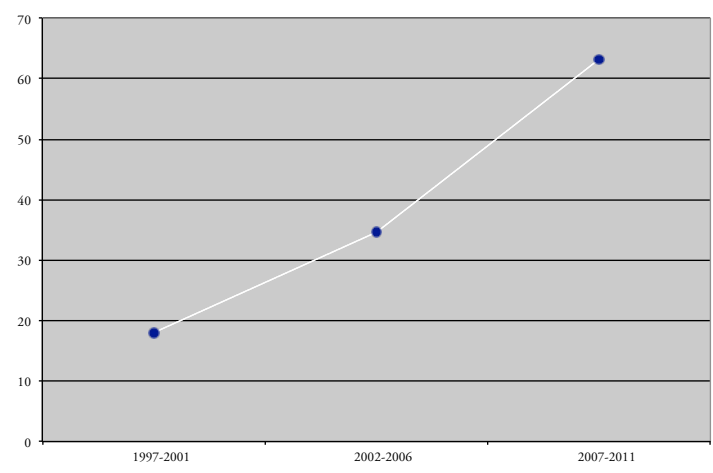

FUENTE ARCHIVOS DE LA REVISTA EST I 997-20 I I

CUADRO 9 | Documentos realizados con colaboración entre instituciones

\begin{tabular}{|l|c|c|c|c|}
\hline \multirow{2}{*}{ PERIODO } & \multicolumn{4}{|c|}{ DOCUMENTOS EN COLABORACIÓN (INSTITUCIONES) } \\
\cline { 2 - 5 } & I INSTITUCIÓN & 2 INSTITUCIONES & 3 INSTITUCIONES & TOTAL \\
\hline $1997-2001$ & 51 & 9 & 2 & 62 \\
\hline $2002-2006$ & 51 & 19 & 6 & 76 \\
\hline $2007-2011$ & 53 & 52 & 19 & 124 \\
\hline Total & $\mathbf{1 5 5}$ & $\mathbf{8 0}$ & $\mathbf{2 7}$ & 262 \\
\hline
\end{tabular}

FUENTE ARCHIVOS DE LA REVISTA EST I 997-20 I I

GRÁFICO 4 | Colaboración entre instituciones

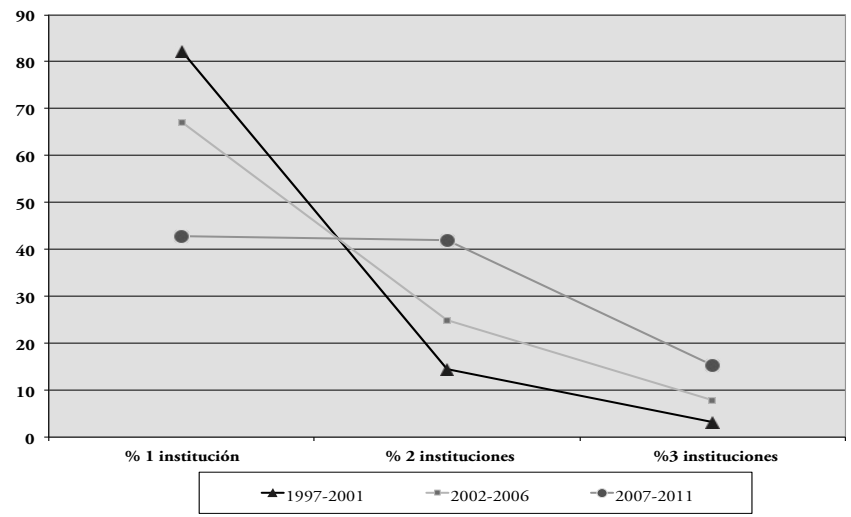

FUENTE ARCHIVO DE LA REVISTA EST I 997-2OI I 


\section{Conclusiones}

Se puede concluir que existen diferencias en la estructura colaborativa de los campos que abarcan los estudios territoriales, las cuales obedecen a las propias tradiciones de las disciplinas asociadas a cada campo, así como de los centros de investigación, y a las motivaciones que encuentren los investigadores para publicar en red.

Por otro lado, se puede apuntar que existe escasa presencia de revistas latinoamericanas de Estudios Urbanos en las bases de datos de corriente principal. Chile es el único país iberoamericano que indiza revistas en todas las bases de datos analizadas, en particular dada la fortaleza de una sola revista de estudios urbanos: EURE. Las bases de datos de acceso abierto muestran una lógica diferente: en el caso de DOAJ (Directory of Open Access Journals), Brasil es el puntero; y en el caso de Redalyc, lo es México. Scielo, por su parte, indiza una menor cantidad de revistas de estudios urbano-territoriales, en comparación con las bases de datos analizadas. Para Scielo y Scopus llama la atención la falta de una clasificación específica por áreas disciplinares (sJR, 2013; Scielo 2013).

En este documento se han presentado las pautas y tendencias de la colaboración en los estudios territoriales; sin embargo, resta conocer la complejidad de dichas estructuras o redes, así como la solidez de sus lazos. Una investigación cualitativa permitiría conocer las pautas de trabajo colaborativo de redes temáticas, equipos de investigación, cuerpos académicos, etcétera, de tal modo que sea posible saber cómo se construye el conocimiento en red. Una futura investigación podría indagar sobre las estrategias que implementan los investigadores para alcanzar los estándares de productividad que demandan los consejos de ciencia y tecnología a los que pertenecen, como el uso de su capital científico para crear relaciones de publicación en red, ya sea con colegas, estudiantes de grado, redes científicas u otros.

\section{Referencias bibliográficas}

Abrizah, A., Zainab, A. N., Kiran, K. \& Raj R. G. (2013). LIs journals scientific impact and subject categorization: a comparison between Web of Science and Scopus. Scientometrics, 94(2), 721.740. doi: 10.1007/s11192-012-0813-7

Aguado-López, E., Rogel-Salazar, R., Álvarez-Velázquez, A., Muñoz-Eraso J. P. \& López-López W. (2008). Producción científica y redes de colaboración en los procesos editoriales: el caso de Cuadernos de Desarrollo Rural en sus 30 ańos. Cuadernos de Desarrollo Rural, 5(61), 11-39. En http://www.redalyc.org/articulo.oa?id=11713138001

Bornmann, L. \& Leydesdorff, L. (2013). Macro-indicators of citation impacts of six prolific countries: InCites data and the statistical significance of trends. PLoS ONE, 8(2), e56768. doi: 10.1371/journal.pone.0056768

Collazo-Reyes, F., Luna-Morales, M. E. \& Vélez Cuartas, G. (2010). Surgimiento de las prácticas científicas de colaboración en la ciencia mexicana con cobertura en los índices internacionales. Redes. Revista Hispana para el Análisis de Redes Sociales, 19(7), 143-167. En http://revista-redes.rediris.es/html-vol19/vol19_7.htm 
CWTS (Centre for Science and Technology Studies, Leiden University). (2012). Merit, expertise andmeasurement: Monitoring knowledgeflows, new performance indicators, and evaluation cultures in 21st Century science, technology and society. CwTs Research Programme, Leiden University. En www.cwts.nl/pdf/cwts_research_programme_2012-2015.pdf

De Mattos, C. (2013). La investigación urbano-regional y la publicación de revistas en América Latina: Una perspectiva histórica y crítica. Ponencia presentada en el Coloquio Internacional sobre Revistas Cientificas. Retos y perspectivas para América Latina, 29 de mayo, Toluca, México.

Kamalski, J. \& Kirby, A. (2012). Bibliometrics and urban knowledge transfer. Cities, 29(Sup.2), S3-S8. http://dx.doi.org/10.1016/j.cities.2012.06.012

Link, F. (2008). EURE 1970-2008: breve genealogía de su trayectoria. EURE, 34(103) 153168. http://dx.doi.org/10.4067/S0250-71612008000300008

Latíndex. (2014). Sistema Regional de Información en Línea para revistas científicas de América Latina, el Caribe, España y Portugal. Universidad Nacional Autónoma de México. En http://www.latindex.unam.mx

Leydesdorff, L. (2003). Can networks of journal-journal citations be used as indicators of change in the social sciences? Journal of Documentation 59(1), 84-104.

Leydesdorff, L. (2013). Advances in science visualization: social networks, semantic maps, and discursive knowledge. En B. Cronin \& C. Sugimoto (Eds.), Bibliometrics and beyond: Metrics-based evaluation of scholarly research (pp. 1-25). Cambridge, MA: The MIт Press. En http://arxiv.org/ftp/arxiv/papers/1206/1206.3746.pdf

Leydesdorff, L., Park, H. W. \& Wagner, C. (2014). International co-authorship relations in the social science citation index: Is internationalization leading the Network? Journal of the Association for Information Science and Technology, 65(10), 2111-2126. doi: 10.1002/ asi. 23102

Miguel, S., Chinchilla-Rodríguez, Z., González C. \& De Moya-Anegón, F. (2012). Analysis and visualization of the dynamics of research groups in terms of projects and coauthored publications. A case study of library and information science in Argentina. Information Research, 17(3) [En línea/sin folios]. En http://eprints.rclis.org/17694/

Molina, J. L., Muñoz J. M. \& Doménech, M. (2002). Redes de publicaciones científicas: un análisis de la estructura de coautorías. Redes. Revista Hispana para el análisis de redes sociales, 1(3) [En línea/sin folios]. En http://revista-redes.rediris.es/html-vol1/ vol1_3.htm

Ni, C., Sugimoto, C. R. \& Cronin, B. (2013). Visualizing and comparing four facets of scholarly communication: producers, artifacts, concepts, and gatekeepers. Scientometrics, 94(3), 1161-1173. doi: 10.1007/s11192-012-0849-8

Perianes-Rodríguez, A., Olmeda-Gómez, C., \& De Moya-Anegón, F. (2008). Introducción al análisis de redes. El Profesional de la Información, 17(6), 664-669. doi 10.3145/ epi.2008.nov.10

Pino-Díaz, J., Jiménez-Contreras, E., Ruíz-Baños, R. \& Bailón-Moreno, R. (2011). Evaluación de redes tecnocientíficas: la red española sobre áreas protegidas, según la Web of Science. Revista Española de Documentación Cientifica, 34(3), 301-333. doi: 10.3989/ redc.2011.3.804 
Redalyc. (2013). Red de Revistas Científicas de América Latina, el Caribe, España y Portugal. Sistema de Información Científica. Universidad Autónoma del Estado de México. Producción científica/Por país (2005-2011). En http://www.redalyc.org/home.oa

Royuela, V. (2012). Regional science trends through the analysis of the main facts of the $51 \mathrm{st}$ ERSA Conference. Investigaciones Regionales, (24), 13-39. En http://www.redalyc.org/ articulo.oa?id $=28924660002$

Scielo. (2013). Lista de revistas 2013. Scientific Electronic Library Online. En http://www. scielo.org/php/index.php?lang=es

Surinach, J., Duque, J. C., Ramos, R. \& Royuela, V. (2002). La investigación regional en España. Un análisis bibliométrico. Investigaciones Regionales, (1), 107-137. En http:// www.redalyc.org/articulo.oa?id $=28900105$

SJR. (2013). Journal Rankings. Scimago Journal \& Country Rank. En http://www.scimagojr. $\mathrm{com} /$

The Royal Society. (2011). Knowledge, networks and nations: Global scientific collaboration in the 21st century. Londres: The Royal Society. En http://royalsociety.org/policy/projects/ knowledge-networks-nations/report/

Web of Science. (2013). Journal Citation Report. Thompson Reuters. En http://thomsonreuters. com/thomson-reuters-web-of-science/ 

RESEÑAS 